\title{
Soret 扩散对层流火焰的影响
}

\author{
韩旺，陈正 ${ }^{*}$ \\ 北京大学工学院力学与工程科学系, 湍流与复杂系统国家重点实验室, 北京 100871 \\ *E-mail:cz@pku.edu.cn
}

收稿日期: 2015-07-09; 接受日期: 2015-08-21

国家自然科学基金(批准号: 51322602)资助项目

\begin{abstract}
摘要在层流火焰中, 各个组分的质量扩散具有十分重要的作用, 它能够影响甚至控制 火焰传播、点火、熄火等过程。通常由浓度梯度引起的 Fick 扩散是占主导地位的，而由温度 梯度引起的 Soret 扩散则相对较小. 因此, 在理论分析和数值模拟中, 通常只考虑 Fick 扩散 而忽略 Soret 扩散. 但是, 以往的研究结果表明, 对于氢气或大分子碳氢燃料的层流燃烧过 程, 当存在较大温度梯度时, Soret 扩散的影响不可忽略. 本文回顾了 Soret 扩散影响层流火 焰的相关研究, 主要介绍了 Soret 扩散对(1)无拉伸和有拉伸预混火焰的传播速度、点火与熄 火, (2)扩散火焰的结构与熄火, 以及(3)喷雾燃烧和碳烟生成的影响.
\end{abstract}

\section{关键词}

Soret 扩散

层流预混火焰

层流扩散火焰

\section{1 引言}

当混合物中存在两种或两种以上化学组分时, 小分子会向高温区扩散而大分子则向低温区扩散. 这种由温度梯度导致的质量扩散被称为 Soret 扩散. Soret 扩散在 1856 年首先被 Lugwig 发现, 在 1879 年 被 Soret 通过实验验证 ${ }^{[1]}$. 由于 Soret 扩散的驱动力是 温度梯度, 因此又被称为热扩散. 在液/固相中, 一般 被称为 Soret 或 Ludwig-Soret 效应(Soret/Ludwig-Soret effect $)^{[2]}$. 在气相中, 一般被称为热泳 (Hermophoresis) ${ }^{[2]}$.

在燃烧过程中, 各个组分的质量扩散包括 Fick 扩散和 Soret 扩散: 由浓度梯度引起的 Fick 扩散是主 要的, 而由温度梯度引起的 Soret 扩散则相对较小. 一般情况下, 在理论分析 ${ }^{\left[{ }^{[25]}\right.}$ 和数值模拟 ${ }^{[6 \sim 8]}$ 中通常 忽略 Soret 扩散而只考虑 Fick 扩散. 但是, 如果存在 非常重或者非常轻的组分和较大的温度梯度, Soret 扩散可能会对层流火焰有较大的影响 ${ }^{[9]}$. 由于燃烧涉
及到多种组分以及较大的温度梯度, 因此有必要研 究 Soret 扩散对燃烧过程的影响. 在湍流燃烧中, 由 于湍流输运导致的质量扩散远大于 Fick 扩散和 Soret 扩散, Soret 扩散的影响可以忽略. 因此本文只关注 Soret 扩散对层流火焰的影响.

在最近四十年, Soret 扩散对燃烧过程的影响已 经被广泛研究 ${ }^{[2,10 ~ 43]}$, 近几年, 本文作者及其同事也对 Soret 扩散做了相应的基础理论与数值研究 ${ }^{[37,38,42,43]}$. 在有关层流火焰的综述文章中 ${ }^{[40,41,44]}$, 都有专门的一 小节用来探讨 Soret 扩散对层流火焰动力学的影响. 在这些综述文章中呈现的结果主要来自数值模拟, 一般只是针对某种特定燃料进行分析. 例如, 文献 [40]总结了 Soret 扩散对氢气火焰的影响; 文献[41]主 要回顾了 Soret 扩散对氢气与甲烷火焰的影响. 据作 者所知, 目前尚无专门回顾与总结 Soret 扩散影响层 流火焰的综述文章. 因此, 本文将介绍和总结 Soret 扩散影响层流火焰的相关研究进展.

引用格式: 韩旺, 陈正. Soret 扩散对层流火焰的影响. 中国科学: 技术科学, 2015, 45: 1117-1129

Han W, Chen Z. Effects of Soret diffusion on laminar flames (in Chinese). Sci Sin Tech, 2015, 45: 1117-1129, doi: 10.1360/N092015-00221 
本文的结构如下: 首先在第 2 和 3 部分分别给出 了数值与理论分析模型; 接着在第 4 和 5 部分分别回 顾了 Soret 扩散对层流预混和扩散火焰的影响; 第 6 部分介绍了 Soret 扩散对喷雾燃烧和碳烟生成的影响; 最后是总结与展望.

\section{2 数值分析模型}

描述燃烧过程的控制方程包括流体动力学方程 (连续性方程、动量方程)、输运方程(组分方程、能量 守恒方程) 以及混合气体的状态方程. 在输运方程中 会涉及动量、能量和组分输运. 另外, 还需要考虑各 个组分之间的化学反应.

\section{1 控制方程}

连续性方程、动量方程、组分方程和能量守恒方 程分别为 ${ }^{[9,45]}$

$$
\begin{gathered}
\frac{\partial \rho}{\partial t}+\nabla \cdot(\rho \boldsymbol{v})=0, \\
\frac{\partial(\rho \boldsymbol{v})}{\partial t}+\nabla \cdot(\rho \boldsymbol{v} \boldsymbol{v})=-\nabla p+\nabla \cdot P+\rho \sum_{k=1}^{N_{S}} Y_{k} \boldsymbol{f}_{k}, \\
\frac{\partial\left(\rho Y_{k}\right)}{\partial t}+\nabla \cdot\left(\rho Y_{k} \boldsymbol{v}\right)=-\nabla \cdot\left(\rho Y_{k} \boldsymbol{V}_{k}\right)+\omega_{k}, \\
\text { for } k=1,2, \cdots, N_{S}, \\
\frac{\partial(\rho h)}{\partial t}+\nabla \cdot(\rho h \boldsymbol{v})=\frac{D p}{D t}+\Phi-\nabla \cdot \boldsymbol{q}+\rho \sum_{k=1}^{N S} Y_{k} \boldsymbol{f}_{k} \boldsymbol{V}_{k},
\end{gathered}
$$

其中, $\boldsymbol{P}$ 和 $\Phi$ 分别是黏性应力张量和黏性耗散:

$$
\begin{aligned}
& \boldsymbol{P}=\mu\left[\nabla \boldsymbol{v}+(\nabla \boldsymbol{v})^{\mathrm{T}}\right]+\left[\left(\kappa-\frac{2}{3} \mu\right)(\nabla \cdot \boldsymbol{v})\right] \boldsymbol{I}, \\
& \Phi=\frac{1}{2} \mu\left(\frac{\partial v_{i}}{\partial x_{j}}+\frac{\partial v_{j}}{\partial x_{i}}\right)^{2}+\left(\kappa-\frac{2}{3} \mu\right)(\nabla \cdot \boldsymbol{v})^{2},
\end{aligned}
$$

$\mu$ 是混合物动力学黏性系数, 采用 Stokes 假设, 可以 忽略体积黏性(即 $\kappa=0$ ).

能量守恒方程中, 焓 $h$ 由生成焓 $h_{k}^{0}$ 和显焓两部 分组成:

$$
h=\sum_{K=1}^{N_{S}} Y_{k} h_{k}^{0}+\int_{T_{0}}^{T} C_{p} \mathrm{~d} T .
$$

理想气体的状态方程为

$$
p=\rho R^{0} T \sum_{k=1}^{N_{s}} \frac{Y_{k}}{W_{k}}=\rho R^{0} T / \bar{W},
$$

其中 $R^{0}$ 是通用气体常数 $(8.314 \mathrm{~J} /(\mathrm{mol} \mathrm{K})), W_{k}$ 是组分 $k$ 的分子量, $\bar{W}$ 是混合物的平均分子量.

\section{2 扩散速度和反应模型}

在方程(3)和(4)中, $\boldsymbol{V}_{k}, \omega_{k}$ 和 $\boldsymbol{q}$ 分别为组分 $k$ 的扩 散速度、化学反应速率和热流通量, 下面给出这些量 的详细模型.

\subsection{1 扩散速度}

组分扩散速度由如下 Stefan-Maxwell 方程确 定 $^{[45]}$ :

$$
\begin{gathered}
\nabla X_{i}=\sum_{j=1}^{N S}\left(\frac{X_{i} X_{j}}{D_{i, j}}\right)\left(\boldsymbol{V}_{j}-\boldsymbol{V}_{i}\right)+\left(Y_{i}-X_{i}\right)\left(\frac{\nabla p}{p}\right) \\
+\left(\frac{\rho}{p}\right) \sum_{j=1}^{N_{S}} Y_{i} Y_{j}\left(\boldsymbol{f}_{i}-\boldsymbol{f}_{j}\right) \\
+\sum_{j=1}^{N S}\left(\frac{X_{i} X_{j}}{\rho D_{i, j}}\right)\left(\frac{D_{T, j}}{Y_{j}}-\frac{D_{T, i}}{Y_{i}}\right)\left(\frac{\nabla T}{T}\right), \\
i=1, \cdots, N_{S},
\end{gathered}
$$

其中, $X_{k}=Y_{k} \bar{W} / W_{k}$ 是组分 $k$ 的摩尔百分数; (9)式右边 第一项代表由浓度梯度驱动的组分扩散(Fick 扩散), $D_{i j}$ 是组分 $i$ 和 $j$ 的二元扩散系数; 第二项代表由压力 梯度驱动的组分扩散; 第三项代表由体力驱动的组 分扩散; 第四项中代表由温度梯度驱动的组分扩散 (Soret 扩散), $D_{T, k}$ 是组分 $k$ 的 Soret 扩散系数. 通常由 压力梯度与体力引起的组分扩散远小于 Fick 扩散和 Soret 扩散, 因此在计算中可以忽略.

\subsection{2 化学反应模型}

考虑如下由 $N_{S}$ 个组分、 $N_{R}$ 个反应组成的多组分 反应流:

$$
\sum v_{k, j}^{\prime} A_{k} \Leftrightarrow \sum v^{\prime \prime}{ }_{k, j} A_{k}, \text { for } j=1, \cdots, N_{R},
$$

其中, $A_{k}$ 代表组分 $k$ 的分子式, $v^{\prime}{ }_{k, j}$ 和 $v^{\prime \prime}{ }_{k, j}$ 表示组分 $k$ 在反应 $j$ 中的反应系数. 于是组分 $k$ 的净生成速 率为

$$
\omega_{k}=W_{k} \sum_{j=1}^{N_{R}}\left(v^{\prime \prime}{ }_{k, j}-v_{k, j}^{\prime}\right) \Psi_{j},
$$

其中, $\Psi_{j}$ 为反应 $j$ 的反应速率:

$$
\Psi_{j}=K_{f, j} \Pi_{k=1}^{N_{S}}\left(\frac{\rho Y_{k}}{W_{k}}\right)^{v_{k, j}^{\prime}}-K_{r, j} \Pi_{k=1}^{N_{S}}\left(\frac{\rho Y_{k}}{W_{k}}\right)^{v^{\prime \prime}, j},
$$

其中, $K_{f, j}$ 和 $K_{r, j}$ 分别是反应 $j$ 的正逆反应速率常数. 


\subsection{3 热流通量}

方程(4)中的热流通量 $\boldsymbol{q}$ 为

$$
\begin{aligned}
\boldsymbol{q}= & -\lambda \nabla T+\rho \sum_{k=1}^{N S} h_{k} Y_{k} \boldsymbol{V}_{\boldsymbol{k}} \\
& +R^{0} T \sum_{i=1}^{N S} \sum_{j=1}^{N S}\left(\frac{X_{j} D_{T, i}}{W_{i} D_{i, j}}\right)\left(\boldsymbol{V}_{i}-\boldsymbol{V}_{j}\right)+\boldsymbol{q}_{R},
\end{aligned}
$$

其中, 右边第一项代表由温度梯度驱动的热量扩散; 第二项代表由组分扩散引起的热量扩散; 第三项代 表由浓度梯度驱动的热量扩散(Dufor 扩散); 第四项 代表体积辐射热损失. 通常 Dufor 扩散非常小 ${ }^{[13]}$, 因 此在计算中也可以忽略.

通过数值求解上述控制方程及相关扩散与反应 模型, 可以模拟层流燃烧过程 ${ }^{[8]}$. 在数值模拟中可以 考虑或者不考虑 Soret 扩散, 对比两种情况下的数值 模拟结果则可以研究 Soret 扩散对层流火焰的影响. 对于本文中涉及的平面火焰、球形传播火焰和对冲火 焰, 可以分别用 CHEMKIN-PREMIX ${ }^{[46]}$, ASURF ${ }^{[47 \sim 50]}$ 和 CHEMKIN-OPPDIF ${ }^{[51]}$ 程序进行模拟.

\section{3 理论分析模型}

本文作者通过理论分析 ${ }^{[42,43]}$ 研究了 Soret 扩散对 预混火焰的影响. 在绝热、低马赫数、常密度(忽略热 膨胀)、常物性、单步不可逆反应假设下, 流体动力学 方程与输运方程解耦, 可以认为燃烧放热不会影响 流场. 对于初始静止的贫燃预混物点火问题, 可以用 一维球形火焰(如图 1(a))传播来近似, 这时控制方程 可以简化为 ${ }^{[42,52,53]}$

$$
\begin{aligned}
\rho C_{P} \frac{\partial T}{\partial t} & =\frac{1}{r^{2}} \frac{\partial}{\partial r}\left(r^{2} \lambda \frac{\partial T}{\partial r}\right)+q \omega, \\
\rho \frac{\partial Y}{\partial t} & =-\frac{1}{r^{2}} \frac{\partial}{\partial r}\left(r^{2} j_{Y}\right)-\omega,
\end{aligned}
$$

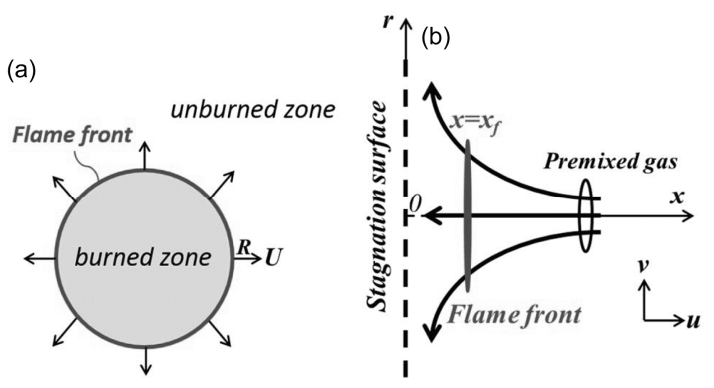

图 1 预混球形传播火焰(a)和对冲火焰(b)
在相同假设下, 对于贫燃预混对冲火焰(如图 $1(b))$, 控制方程可以简化为 ${ }^{[43]}$

$$
\begin{gathered}
\rho C_{P} u \frac{\mathrm{d} T}{\mathrm{~d} x}=\frac{\mathrm{d}}{\mathrm{d} x}\left(\lambda \frac{\mathrm{d} T}{\mathrm{~d} x}\right)+q \omega, \\
\rho u \frac{\mathrm{d} Y}{\mathrm{~d} x}=-\frac{\mathrm{d}}{\mathrm{d} x}\left(j_{Y}\right)-\omega,
\end{gathered}
$$

其中, 单步全局化学反应的速率为

$$
\omega=\rho A Y \exp \left(-\frac{E}{R^{0} T}\right),
$$

在方程(14) (18)中 $T$ 和 $Y$ 分别为混合物温度和燃料质 量百分数; $t$ 和 $r$ 分别为时间与空间坐标; $u=-\kappa x$ 为对 冲火焰轴向 $(x)$ 速度, $\kappa$ 为拉伸率; $\rho$ 为气体密度, $C_{P}$ 为 定压比热, $\lambda$ 为混合物热传导系数; $q$ 为燃烧单位质量 燃烧释放的热量, $A$ 为指前因子, $E$ 为化学反应活化 能.

在理论分析中采用混合物平均扩散模型, 在方 程(15)和(17)中, 质量扩散通量为

$$
j_{Y}=-\rho D \cdot \nabla Y-\alpha \cdot \rho D \cdot Y \nabla \ln (T),
$$

其中, 等式右边第一项代表 Fick 扩散, 第二项代表 Soret 扩散; $\alpha$ 为 Soret 扩散系数, 对于轻分子组分 $\alpha$ 取负值, 对于重分子组分 $\alpha$ 取负值 ${ }^{[9]}$. 对于氢气/空气 混合物, $\alpha_{\mathrm{H}_{2}}=-0.29, \alpha_{\mathrm{H}}=-0.23, \alpha_{\mathrm{H}_{2} \mathrm{O}}=-0.02, \alpha_{\mathrm{O}_{2}}=$ $+0.02^{[40]}$.

\section{Soret 扩散对层流预混火焰的影响}

我们首先介绍 Soret 扩散影响层流预混火焰的相 关研究. 这里考虑三种预混火焰: 无拉伸平面火焰、 对冲火焰和球形传播火焰, 后两种预混火焰均具有 正的拉伸率.

\section{1 无拉伸平面火焰}

层流火焰速度是反映预混燃料燃烧特性的重要 参数. 通过数值模拟一维无拉伸平面火焰可以获得 不同工况下的层流火焰速度. 目前, 文献中已有关于 Soret 扩散如何影响氢气、合成气、甲烷、正丁烷、 正庚烷等燃料层流火焰速度的研究. Dixon-Lewis ${ }^{[10]}$ 首先通过数值模拟研究了 Soret 扩散对氢气/空气平 面无拉伸预混火焰结构的影响, 他发现当不考虑 $\mathrm{H}$ 原子的 Soret 扩散时层流火焰速度会增加. 接着 Greenberg ${ }^{[11]}$ 对氢气/空气平面无拉伸预混火焰进行了 
详细的数值模拟. Ern 和 Giovangigli ${ }^{[19,20]}$ 研究了 Soret 扩散对氢气和甲烷的 Bunsen 火焰的影响. Bongers 和 de Goey ${ }^{[24]}$ 探讨了不同质量输运模型对层流火焰速度 的影响. Yang 等人 ${ }^{[32,33]}$ 研究了 Soret 扩散影对氢气和 正丁烷平面火焰和层流火焰速度的影响. Xin 等人 ${ }^{[35]}$ 研究了 Soret 扩散对庚烷燃烧的影响. Liang 等人 ${ }^{[37]}$ 分析了Soret 扩散影响合成气的扩散与化学反应机制.

图 2 给出了氢气/空气和正丁烷/空气混合物的层 流火焰速度随当量比变化情况. 其中实线和虚线分别 为考虑和不考虑 Soret 扩散时所对应的结果. 可以看 出, 对于氢气/空气混合物, Soret 扩散对层流火焰速 度具有较大的影响. 而对于正丁烷这样的大分子燃 料, Soret 扩散对其层流火焰速度的影响则可以忽略.

Liang 等人 ${ }^{[37]}$ 研究了 Soret 扩散对合成气 $\left(\mathrm{H}_{2} / \mathrm{CO}\right)$ 层流火焰速度的影响. 图 3 给出了不同氢气与一氧化 碳比例下层流火焰速度随当量比的变化情况. 可以
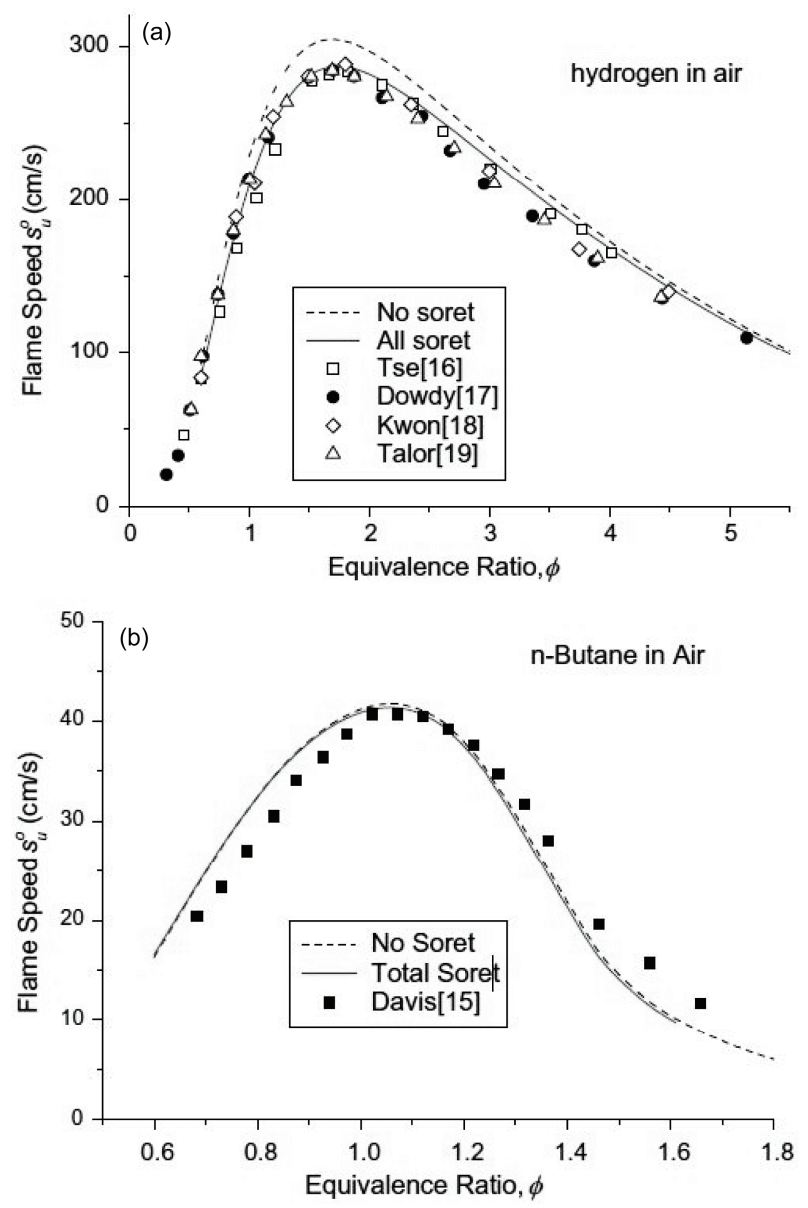

图 2 氢气/空气(a)和正丁烷/空气(b)混合物的层流火焰速度 随当量比的变化情况 $\left(T_{u}=298 \mathrm{~K}, P=1 \mathrm{~atm}\right)^{[33]}$

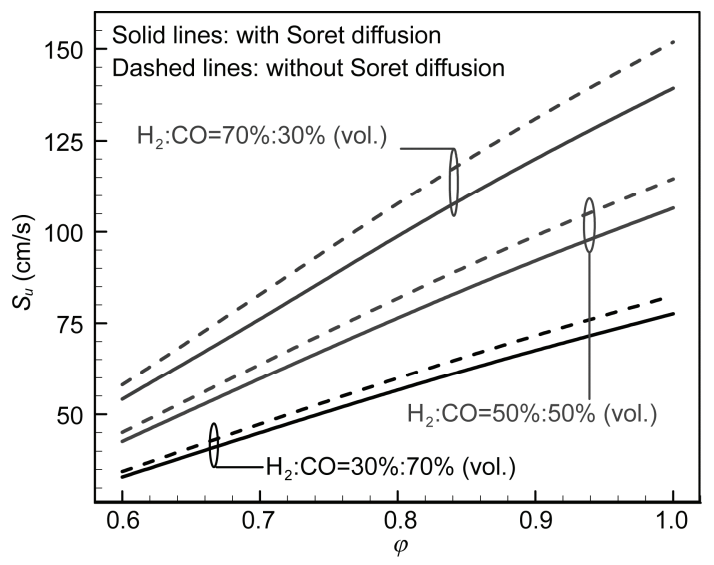

图 3 不同当量比与氢气百分含量下合成气的层流火焰速 度 $\left(T_{u}=298 \mathrm{~K}, P=1 \mathrm{~atm}\right)^{[37]}$

看出氢气百分比越大, 相应的层流火焰速度受 Soret 扩散的影响越大. 另外, 随着当量比增大, Soret 扩散 对于层流火焰速度的影响也增强. 当量比和氢气百 分比通过 Soret 扩散对层流火焰速度的影响可以解释 为: 合成气的 Soret 扩散主要是 $\mathrm{H}_{2}$ 和 $\mathrm{H}$ 自由基这两个 轻质组分造成的; 在当量比较大或者氢气百分比较 大的情况下, 轻质组分所占的比例也较大, 使得 Soret 对层流火焰速度的影响更为明显.

图 2 和 3 均为常温常压的结果, 接下来将讨论不 同温度和压力下的结果. 图 4 给出了不同温度和压强 下考虑和不考虑 Soret 扩散时的合成气层流火焰速度 计算结果. 可以看出, 压强越小, Soret 扩散对层流火 焰速度造成的影响越大; 而在 $25 \mathrm{~atm}$ 的高压情况下, Soret 扩散几乎不会对层流火焰速度造成影响. 而且, 在不同的压力下 Soret 扩散对于层流火焰速度的影响 都会随着温度上升而增强. 注意到一维平面火焰的 计算中, 求解的特征值是燃烧质量流量 $m=\rho_{u} S_{u}$ 而不 是层流火焰速度 $S_{u}{ }^{[9]}$, 图 4 进一步比较了不同压力与 初始温度下的燃烧流量. 可以看出, 压力或者温度越 高, 则 Soret 扩散对燃烧流量的影响越显著. 因此, 对于高温高压下的合成气燃烧过程, 需要考虑 Soret 扩散的影响.

为了区分 $\mathrm{H}$ 自由基和 $\mathrm{H}_{2}$ 的 Soret 扩散在不同压 力下的影响, 图 5 给出了由于 $\mathrm{H}$ 自由基的 Soret 扩散、 $\mathrm{H}_{2}$ 的 Soret 扩散、 $\mathrm{H}$ 自由基和 $\mathrm{H}_{2}$ 的 Soret 扩散以及全 体组分的 Soret 扩散导致的燃烧质量流量 $m$ 的改变量 $\delta m$. 可以看出仅考虑 $\mathrm{H}$ 自由基和 $\mathrm{H}_{2}$ 的 Soret 扩散得 到的结果与考虑全体组分 Soret 扩散得到的结果几 


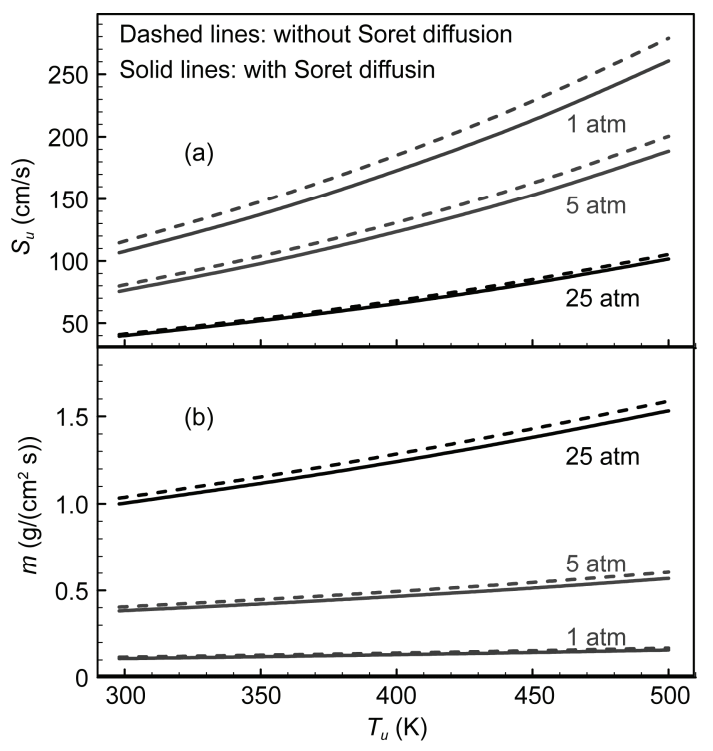

图 4 不同温度和压强下的合成气层流火焰速度与燃烧流 量随初始温度的变化情况 ${ }^{[37]}$

$\mathrm{H}_{2}: \mathrm{CO}=50 \%: 50 \%$ vol., 当量比为 1.0
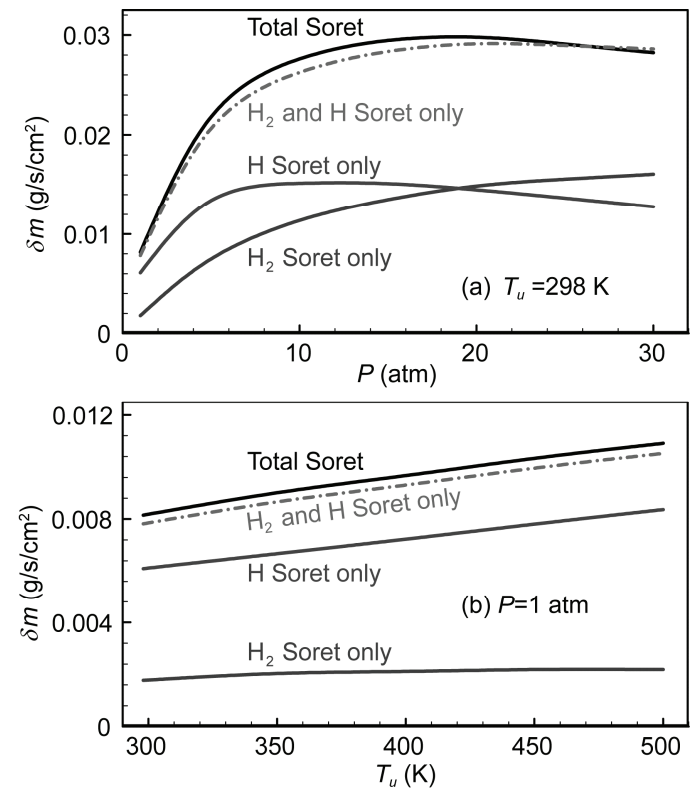

图 5 燃烧质量流量 $m$ 的改变量 $\delta m$ 随初始压强(a)和初始温 度(b)的变化关系图 ${ }^{[37]}$

乎一样. 因此, 可以认为只有 $\mathrm{H}$ 自由基和 $\mathrm{H}_{2}$ 的 Soret 扩散起主要作用. 尽管在常压时, 整体的 Soret 扩散主 要是由 $\mathrm{H}$ 自由基的 Soret 扩散引起的, 然而压力增加 后 $\mathrm{H}_{2}$ 的 Soret 扩散变得越来越重要, 并且在 $P>20$ atm 时超过 $\mathrm{H}$ 自由基 Soret 扩散的影响. 随着温度的升高,
$\mathrm{H}$ 自由基和 $\mathrm{H}_{2}$ 的 Soret 扩散的影响也越来越重要.

为了解释图 4 中的结果, 我们考虑在合成气的化 学反应机理中, 影响层流火焰速度的重要基元反应 为 $\mathrm{H}_{2}-\mathrm{O}_{2}$ 系统的链分支和链传递反应 $\mathrm{R} 1: \mathrm{H}+\mathrm{O}_{2} \rightarrow$ $\mathrm{OH}+\mathrm{O} ; \mathrm{R} 2: \mathrm{H}_{2}+\mathrm{O} \rightarrow \mathrm{H}+\mathrm{OH} ; \mathrm{R} 3: \mathrm{H}_{2}+\mathrm{OH} \rightarrow \mathrm{H}+\mathrm{H}_{2} \mathrm{O}$. 图 6 给出了上述基元反应的反应速率. 可以看出 Soret 扩散使得上述几个反应的反应速率降低, 从而减小 了总体的反应速率. 由于 Soret 扩散影响层流火焰速 度的主要原因是削弱化学反应, 可以通过主反应区 的位置来判断 Soret 扩散的影响强弱.

由于在化学反应与 Soret 扩散流量耦合的区间 Soret 扩散才会对火焰传播有明显影响, 图 7 比较了 不同压力与初始温度条件下 Soret 扩散流量关于温度 的分布. 为了显示化学反应的主要作用区间, 选取 $H$ 自由基的特征反应 $\mathrm{R}_{1}$ 和 $\mathrm{H}_{2}$ 的特征反应 $\mathrm{R}_{3}$, 将其化学 反应速率大于峰值的 $50 \%$ 的温度区间在图中标出. 可以看出, $\mathrm{H}$ 的 Soret 扩散流量峰值恰好位于主反应 区之间, 而 $\mathrm{H}_{2}$ 的 Soret 扩散流量峰值则偏向主反应区 左侧的低温区. 因此, $\mathrm{H}$ 的 Soret 扩散起主导作用, 而 氢气的 Soret 扩散对合成气火焰速度影响不大, 这与 Yang 等人 ${ }^{[32]}$ 验证的 $\mathrm{H}_{2}$ 层流火焰的结果相同.

虽然 Soret 扩散并不会影响平面无拉伸火焰的绝 热火焰温度(因为不影响焓守恒), 但是会改变自由基 的输运, 影响火焰结构与化学反应速率(图 7), 从而 影响层流火焰速度. 而且这种影响会随着当量比, 初 始温度和初始压力不同而变化.

\section{2 拉伸火焰}

在实际燃烧过程中, 由于曲率和流场非均匀性

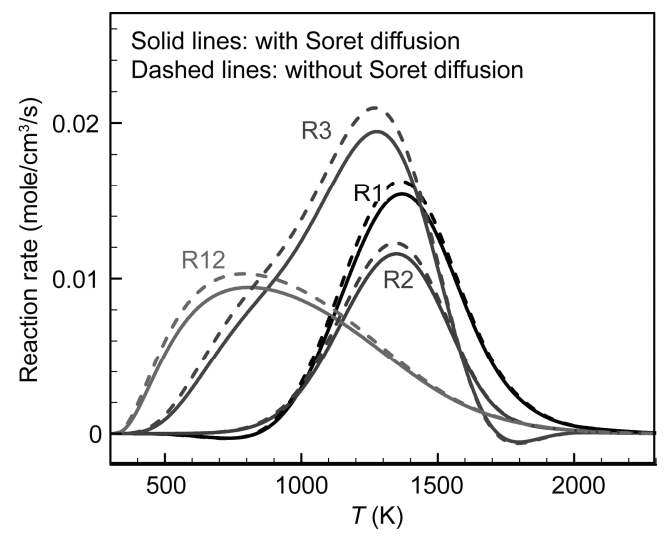

图 6 基元反应的反应速率 ${ }^{[37]}$

$\mathrm{H}_{2}: \mathrm{CO}=50 \%: 50 \%$ vol., 当量比为 $1.0, T_{u}=298 \mathrm{~K}, P=1 \mathrm{~atm}$ 


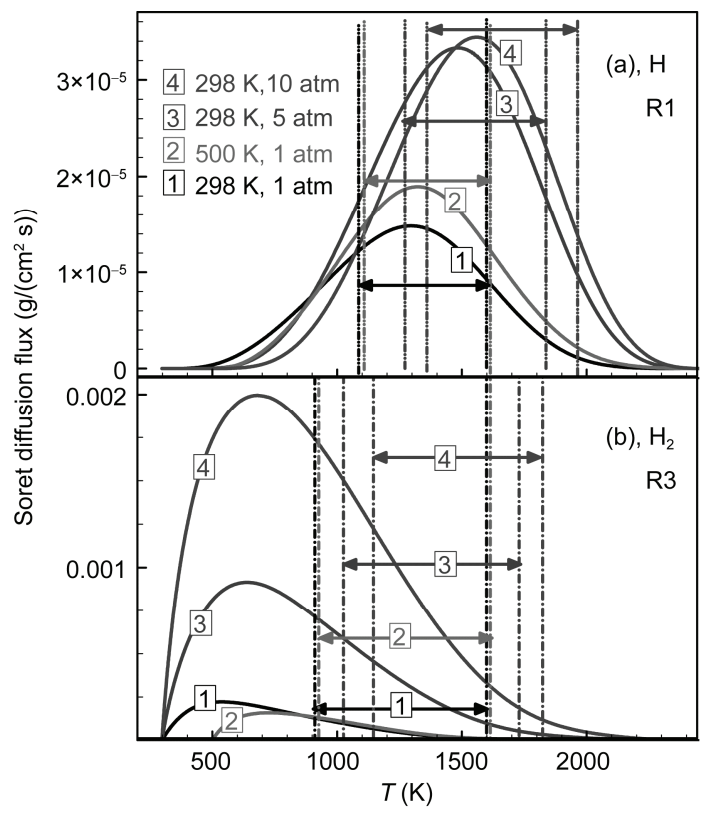

图 $7 \mathrm{H}$ 和 $\mathrm{H}_{2}$ 的 Soret 扩散流量与主反应区的耦合

$\mathrm{H}_{2}: \mathrm{CO}=50 \%: 50 \%$ vol., 当量比为 $1.0^{[37]}$

会导致火焰面受到拉伸率的影响. 火焰拉伸与 Lewis 数 (定义为热扩散系数与质量扩散系数的比值)相结 合会影响火焰的强弱 ${ }^{[9,54]}$. 通常基于球形传播火焰与 对冲火焰来研究 Soret 扩散对拉伸火焰的影响. Garcia-Ybarra 等人 ${ }^{[12,13]}$ 揭示了 Soret 扩散对拉伸预混 火焰的影响; Ern 和 Giovangigli ${ }^{[19,20]}$ 研究了 Soret 扩散 对氢气对冲火焰的影响; Yang 等人 ${ }^{[32,33]}$ 研究了 Soret 扩散影对氢气预混对冲火焰的影响; Xin 等人 ${ }^{[35]}$ 研究 了 Soret 扩散对正庚烷预混对冲火焰的影响; Zhang 等人 ${ }^{[36]}$ 探讨了 Soret 扩散对正丁烷预混对冲火焰的 影响; Liang 等人 ${ }^{[37,38]}$ 研究了 Soret 扩散影响合成气的 球形火焰点火与传播机制. 最近, Han 和 Chen ${ }^{[42,43]}$ 分 析了 Soret 扩散对预混球形火焰点火与传播的影响以 及对预混对冲火焰的熄火拉伸率的影响.

我们通过球形传播火焰来分析了 Soret 扩散对预 混物点火与火焰传播的影响 ${ }^{[42]}$. 采用准稳态假设及 渐近分析求解方程(14)和(15), 可以得到描述球形火 焰传播速度与火焰半径的代数关系式, 具体的无量 纲过程与求解方法读者可以参考文献[42]. 图 8 给出 了无量纲火焰传播速度随无量纲火焰半径变化曲线. 从图 8 中可以看出, 对于轻质燃料 $(L e=0.5)$, Soret 扩 散会增加火焰传播速度, 而对于大分子燃料 $(L e=2.0)$, Soret 扩散会降低火焰传播速度. 这主要是由于 Soret 扩散会驱动小分子燃料向高温反应区扩散从而增加

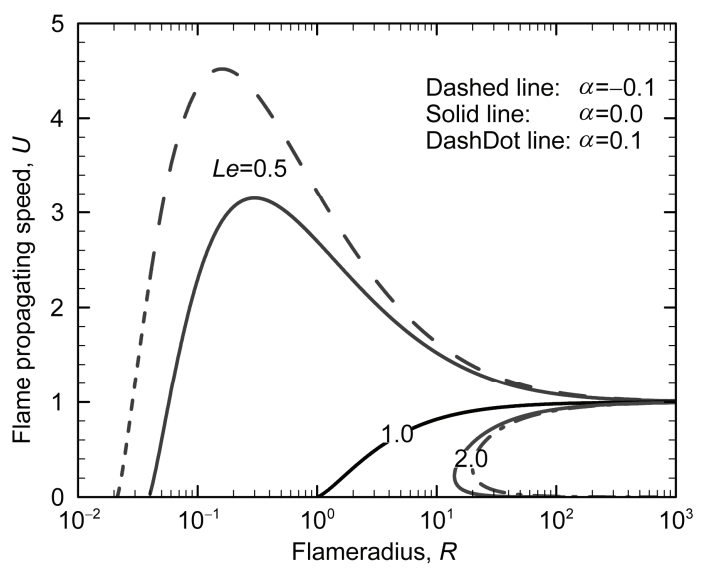

图 8 无量纲火焰传播速度随无量纲火焰半径的变化 ${ }^{[42]}$

反应区的局部当量比, 而大分子燃料在 Soret 扩散作 用下会向低温区扩散从而减小了反应区的局部当量 比. 从图 8 中还可以发现, 在火焰半径较小时, Soret 扩散的作用越发明显, 因此可以推测 Soret 扩散对球 形火焰的点火过程有显著的影响.

Markstein 数表征了火焰传播速度对火焰拉伸率 的变化的敏感性. 图 9 给出了相对 Markstein 长度(定 义为 $L(\alpha) / L(\alpha=0))$ 随 Soret 扩散系数的变化. 当不考 虑 Soret 扩散时：对于轻质燃料 $(L e=0.5), L(\alpha=0)=-4.7$; 对于大分子燃料 $(L e=2.0), L(\alpha=0)=+3.8$. 从图 9 中可 以看出, 对于轻质燃料 $(L e=0.5)$ 与大分子燃料 $(L e=2.0)$, 相对 Markstein 长度随 Soret 扩散系数线性 增加, 这说明当考虑 Soret 扩散时, 球形火焰对拉伸 率的变化越发敏感. 这进一步表明 Soret 扩散会对点 火过程(高拉伸率过程)产生显著的影响.

图 10 给出了合成气火焰传播速度 $S_{b}$ 随着火焰半 径 $R$ 变化的数值模拟结果 ${ }^{[37]}$. 该图表明不同组分的

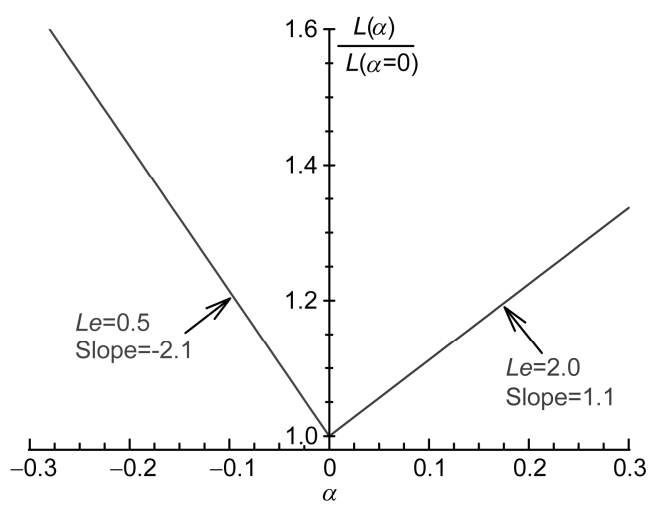

图 9 相对 Markstein 数随 Soret 扩散系数的变化 ${ }^{[42]}$ 


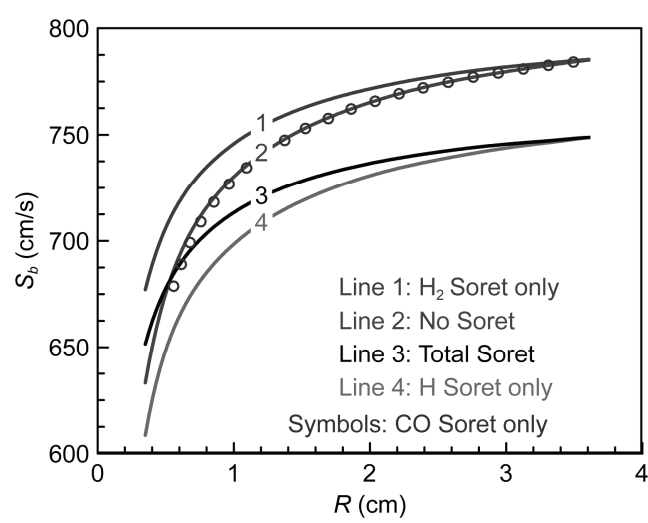

图 10 考虑不同组分 Soret 扩散的火焰速度随火焰半径 变化 ${ }^{[37]}$

$\mathrm{H}_{2}: \mathrm{CO}=50 \%: 50 \%$ vol., 当量比为 $1.0, T_{u}=298 \mathrm{~K}, P=1 \mathrm{~atm}$

Soret 扩散会显著地影响拉伸火焰的传播速度. 由于 球形火焰的火焰拉伸率 $K$ 与火焰半径 $R$ 成反比, 火焰 半径较大时其对应的拉伸率较小, 反之亦然. 在火焰 半径较大时, 由于拉伸率较小, 其火焰传播特性就类 似于无拉伸的平面火焰. 当火焰半径 $R$ 大于 $3 \mathrm{~cm}$ 时, 由图 10 可以看出 Soret 扩散效应降低了火焰速度. 并 且这一效应是由于 $\mathrm{H}$ 自由基的 Soret 扩散造成的, 而 $\mathrm{H}_{2}, \mathrm{CO}$ 和 $\mathrm{OH}$ 等组分 Soret 扩散的影响很小. 因此, 在 常压下拉伸率较小时, Soret 扩散对于拉伸火焰速度 的影响主要是 $\mathrm{H}$ 自由基造成的. 然而对于火焰半径 较小 $(0.5 \mathrm{~cm}<R<1.5 \mathrm{~cm})$, 拉伸率较大的情况, 图 10 表 明火焰传播速度会被 $\mathrm{H}$ 自由基和 $\mathrm{H}_{2}$ 两种组分的 Soret 扩散效应影响, 而 $\mathrm{CO}$ 和 $\mathrm{OH}$ 自由基等组分的 Soret 扩散依然不重要.

图 11 给出了火焰传播速度 $S_{b}$ 随着拉伸率 $K$ 变化 的数值模拟结果. 可以看出在拉伸率较大的情况下, $\mathrm{H}$ 自由基与 $\mathrm{H}_{2}$ 的 Soret 扩散都会对于拉伸火焰速度造 成影响. 然而, 当拉伸率较小时 (如 $K<500 \mathrm{~s}^{-1}$ ), 或者 拉伸率为零时, $\mathrm{H}_{2}$ 的 Soret 扩散效应对于 $S_{b}$ 的影响很 小. 但是与拉伸率较小或者等于零的情况下的火焰 速度不同, $\mathrm{H}$ 自由基的 Soret 扩散对于 Markstein 长度 的影响很小. 然而, $\mathrm{H}_{2}$ 的 Soret 扩散对于 Markstein 长 度影响明显. 这是因为 Markstein 长度对于燃料组分 Lewis 数的敏感性要远大于其对于中间产物的 Lewis 数的敏感性 ${ }^{[53,55]}$. 在压力为 $1 \mathrm{~atm}$ 和初始温度为 298 $\mathrm{K}$ 的工况下, $\mathrm{H}_{2}$ 的 Soret 扩散导致 Markstein 长度的相 对变化为 $32 \%$. 还可以看出考虑所有组分的 Soret 扩 散得出的 Markstein 长度与只考虑 $\mathrm{H}_{2}$ 的 Soret 扩散得

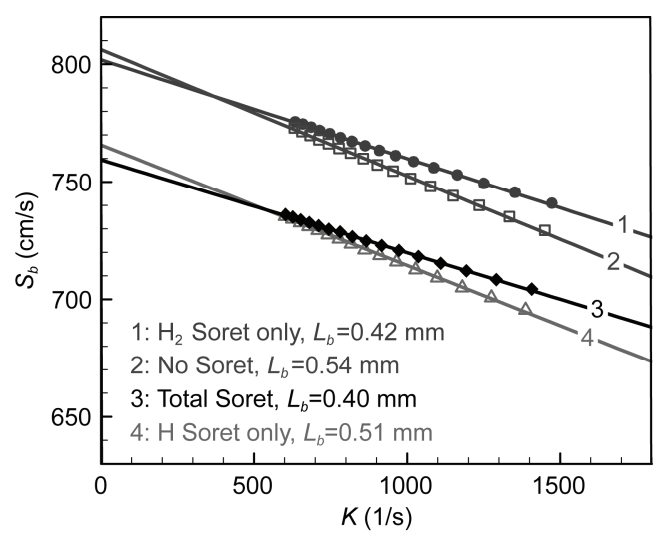

图 11 火焰速度随火焰拉伸率变化 ${ }^{[37]}$

出的结果几乎一样. 因此, 影响 Markstein 长度的主 要是 $\mathrm{H}_{2}$ 的 Soret 扩散效应.

图 12 给出了 Soret 扩散对球形火焰核演化的影 响. 从图中可以发现, 当没有点火能 $Q$ 时, 火焰只有 一个右边的传播分支, 逐渐增加点火能时, 有两个 $U-R$ 分支, 左边的为点火核传播分支, 右边为传播分 支. 当点火能达到最小点火能 $Q_{c}$ 时, 两支相交点火 成功. 图 12 表明, Soret 扩散会定量地影响球形火焰 核的演化轨迹, 不考虑 Soret 扩散时的最小点火能为 1.931, 而考虑 Soret 扩散时的最小点火能为 3.205 , 最 小点火能增加了 $66 \%$. 因此 Soret 扩散会使得大分子 燃料点火变得更加困难.

图 13 给出了相对最小点火能 $Q_{c}(\alpha) / Q_{c}(\alpha=0)$ 随 Soret 扩散系数的变化曲线. 对于轻质燃料 $(L e=0.5)$ 最 小点火能随着 Soret 扩散增强 $(\alpha$ 从 $0 \rightarrow-0.3)$ 而减小, 点火变得容易, 而对于大分子燃料, Soret 扩散 $(\alpha$ 从 0 $\rightarrow+0.3)$ 的存在会增加最小点火能, 从而使点火变的 困难. 文献 [38]采用详细的数值模拟方法研究了 Soret 扩散对氢气/ 空气混合物最小点火能的影响, 数 值模拟结果与理论结果定性上是一致的.

对于拉伸火焰，当拉伸率超过某一临界值时，火 焰就会熄灭 ${ }^{[9]}$, 这个临界拉伸率称为熄火拉伸率 $K_{\text {ext }}$. 最近, 文献[32,35]通过数值模拟分析了 Soret 扩散对 预混对冲火焰熄火拉伸率的影响. 为了得到更加一 般的结论, 文献[43]通过渐近理论分析了 Soret 扩散 对预混对冲火焰的影响. 图 14 给出了通过渐近求解 方程(17)和(18)得到的熄火拉伸率 $K_{\text {ext }}$ 随 Soret 扩散系 数 $\alpha$ 的变化曲线. 图 14 中 $L e=0.5$ 代表轻质燃料, 对 应的 Soret 扩散系数小于 $0 ; L e=2.0$ 代表大分子燃料, Soret 扩散系数大于 0 . 从图 14 中可以发现, 对于轻 

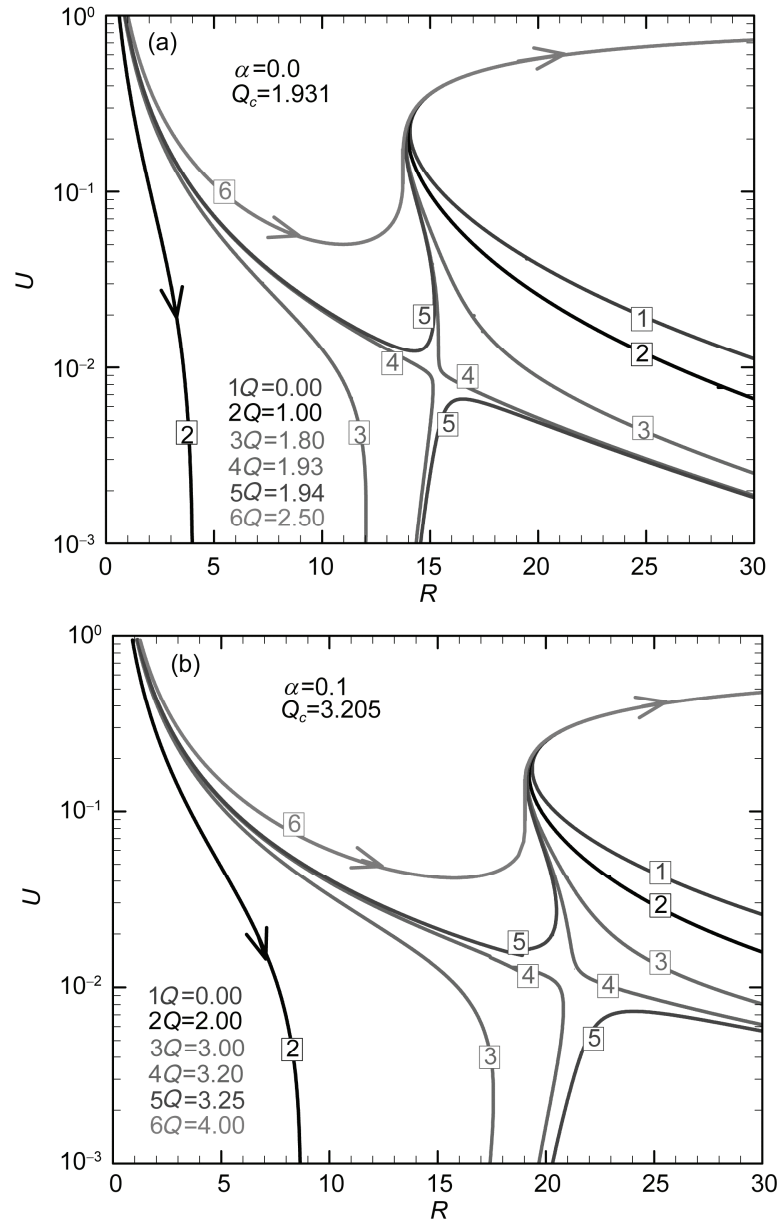

图 12 大分子燃料 $(L e=2.0)$ 的球形点火核随最小点火能的 演化轨迹 ${ }^{[42]}$

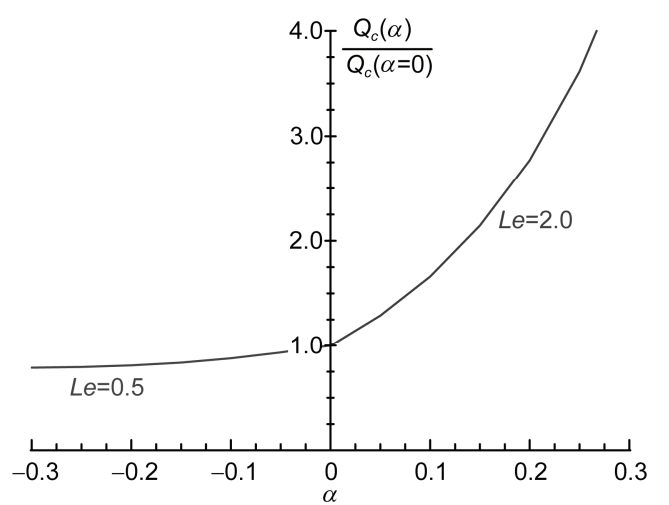

图 13 标准化的最小点火能随 Soret 扩散系数的变化 ${ }^{[42]}$

质燃料, 当考虑 Soret 扩散时 $(\alpha$ 从 $0 \rightarrow-0.3)$, 熄火拉 伸率会增大，火焰更不容易熄火，而对于大分子燃料， Soret 扩散 $(\alpha$ 从 $0 \rightarrow+0.3)$ 的存在会使熄火拉伸率降 低, 火焰更容易熄灭. 这与数值模拟结果定性一致.

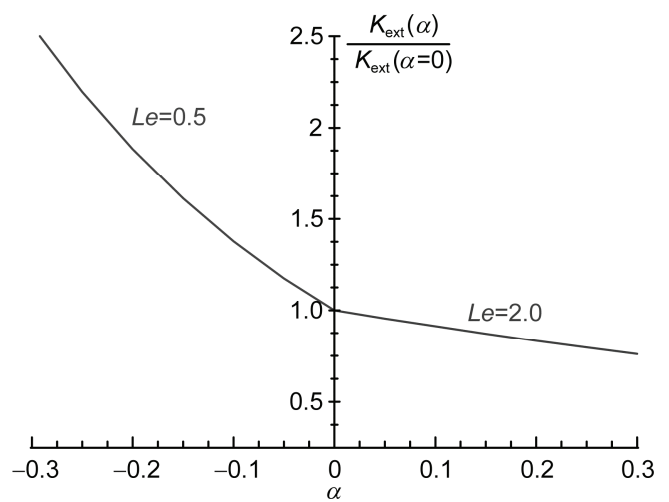

图 14 熄火拉伸率随 Soret 系数的变化 ${ }^{[43]}$

\section{Soret 扩散对层流扩散火焰的影响}

不同于预混火焰，扩散火焰的火焰面处温度最 高而火焰两侧温度较低. 因此, Soret 扩散存在会增加 火焰面处的轻质燃料或自由基的浓度, 降低大分子 燃料的浓度. Williams ${ }^{[22]}$ 分析了 Soret 扩散对对冲甲烷/ 空气扩散火焰的结构和熄火特性的影响. 文献[56]研 究了 Soret 扩散对正庚烷扩散火焰点火机制的影响. 最近, Yang 等人 ${ }^{[32,33,36]}$ 详细探讨了 Soret 扩散对氢气 和正丁烷扩散对冲火焰的影响, Xin 等人 ${ }^{[35]}$ 考察了 Soret 扩散对正庚烷扩散对冲扩散燃烧的影响, Arias-Zugasti 和 Rosner ${ }^{[28]}$ 研究了 Soret 扩散对对冲扩 散火焰温度和位置的影响, Fong 等人 ${ }^{[34]}$ 探讨了 Soret 扩散对对冲扩散火焰的熄火的影响.

图 15 给出了 Soret 扩散对氢气/空气对冲扩散火 焰温度的影响. 可以看出, 当仅考虑 $\mathrm{H}_{2}$ 分子和 $\mathrm{H}$ 自 由基的 Soret 扩散时, 火焰会变强, 尤其在熄火拉伸 率 $K_{\text {ext }}$ 附近, Soret 扩散对火焰温度的影响更加显著, 这是由于在熄火极限附件, 有限化学反应速度将占 主导作用, Soret 扩散会使 $\mathrm{H}_{2}$ 分子和 $\mathrm{H}$ 自由基向高温 区扩散，从而提高反应区的局部当量比和活性自由 基浓度进而加强了火焰，提高了熄火拉伸率.

为了研究 Soret 扩散对大分子燃料扩散火焰的影 响, Xin 等人 ${ }^{[35]}$ 分析了 Soret 扩散对 n-heptane 对冲扩 散火焰的影响, 如图 16. 与大分子燃料预混火焰相 同, 虽然 $\mathrm{H}_{2}$ 分子和 $\mathrm{H}$ 自由基的 Soret 扩散会使反应区 的活性分子浓度增大从而加强火焰, 但 n-heptane 燃 料的 Soret 扩散对火焰的减弱效应是占主导的. 因此 当考虑所有成分的 Soret 扩散时, 火焰强度会变弱从 而降低了熄火拉伸率. 


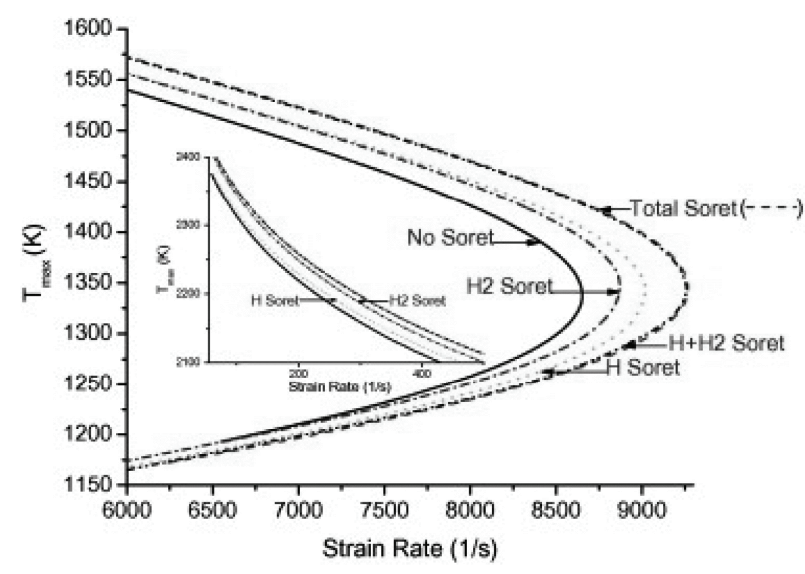

图 15 氢气/空气对冲扩散火焰温度随拉伸率的变化 ${ }^{[32]}$

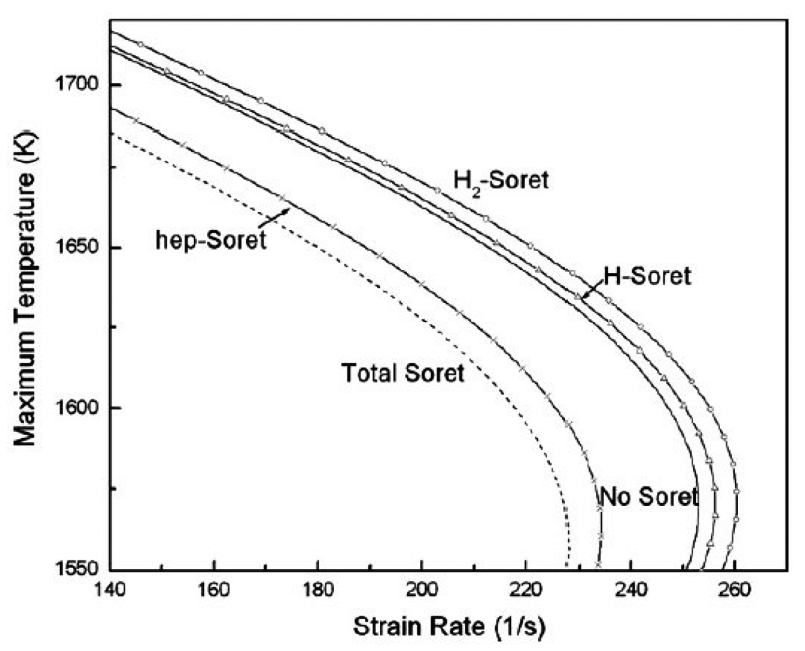

图 $16 \mathrm{C}_{7} \mathrm{H}_{16} /$ 空气对冲扩散火焰温度随拉伸率的变化 ${ }^{[35]}$

Fong 等人 ${ }^{[34]}$ 通过渐近理论分析了 Soret 扩散对 扩散对冲火焰的影响. 图 17 显示了无量纲火焰温度 随剩余燃料质量百分数的变化, 其中 $\alpha_{s}$ 代表燃料 Soret 扩散系数. 从图中可以看出, 火焰温度随着剩 余燃料质量百分数的增加而降低, 而燃料的 Soret 扩 散会修正这个下降趋势. 对于轻质燃料 $\left(\alpha_{s}<0\right)$, 考虑 Soret 扩散时的火焰温度要高于忽略 Soret 时的温度, 尤其在剩余燃料质量百分数较大的时候, 这是由于 在极限情况下, 火焰温度对有限速率的化学反应更 加敏感, Soret 扩散会驱动轻质燃料向火焰区扩散, 增 加反应区局部当量比从而提高火焰温度. 而对大分 子燃料, Soret 扩散会降低反应区的局部当量比从而 降低火焰温度. 这与数值模拟的结果定性上是一 致的.

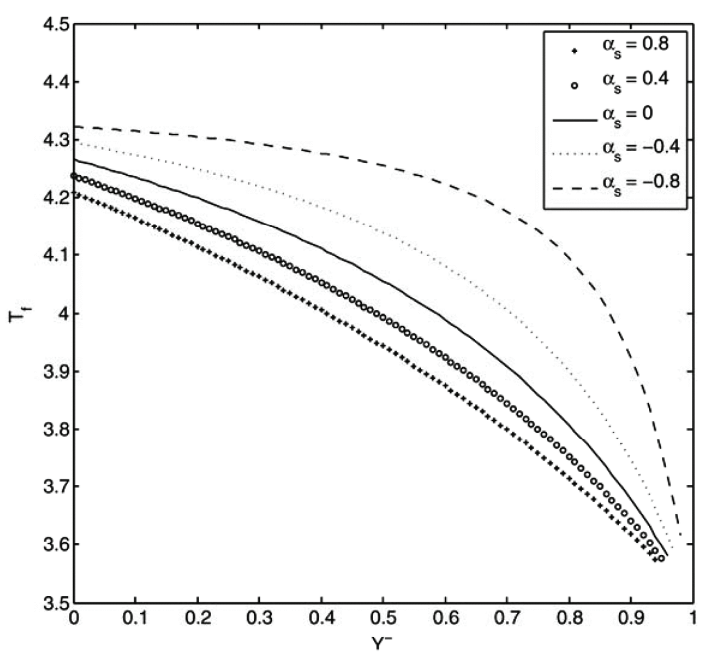

图 17 无量纲火焰温度 $T_{f}$ 随剩余燃料质量百分数 $Y$ 的变 化 ${ }^{[34]}$

\section{Soret 扩散对喷雾燃烧和碳烟生成的影响}

喷雾燃烧广泛存在于实际燃烧设备中，在液滴 蒸发过程中, 气相的扩散作用对火焰结构与动力学 有较大的影响. Dakhlia 等人 ${ }^{[23]}$ 研究了 Soret 扩散对甲 醇对冲扩散喷雾火焰的影响. 图 18 给出了 Soret 扩散 对甲醇喷雾燃烧中 $\mathrm{H}_{2}$ 摩尔百分数的影响. 其中实线 代表精确扩散速度模型(包含 Soret 扩散), 虚线代表 精确扩散速度模型(不包含 Soret 扩散), 点线代表近 似扩散速度模型(不包含 Soret 扩散). 从图中可以发 现当采用精确扩散速度模型并考虑 Soret 扩散时, $\mathrm{H}_{2}$ 分子会向高温区扩散而参与反应, 所以 $\mathrm{H}_{2}$ 分子摩尔 百分数相对较低.

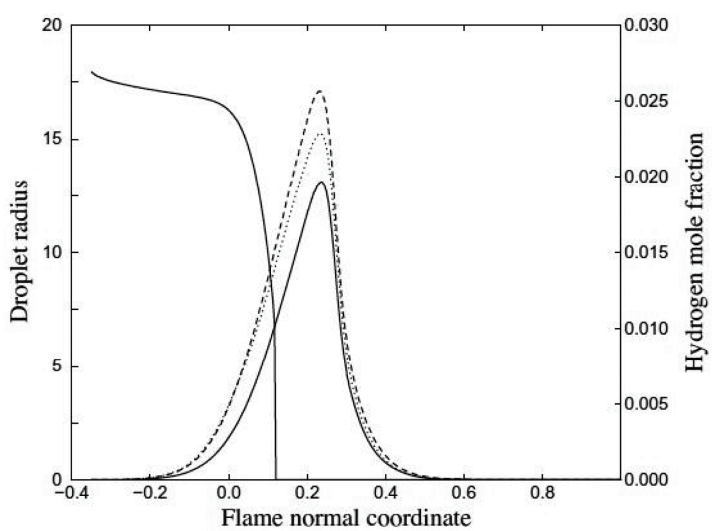

图 18 甲醇喷雾对冲扩散火焰: 液滴半径 $(\mu \mathrm{m})$ 和 $\mathrm{H}_{2}$ 摩尔百 分数随空间位置的变化 ${ }^{[23]}$ 
为了研究 Soret 扩散对液滴蒸发模型的影响, 文 献[23]给出了考虑气相 Soret 扩散时的液滴蒸发模型. 图 19 显示了 Soret 扩散对甲醇摩尔百分数的影响. 其 中实线表示考虑液滴蒸发模型中的气相 Soret 扩散, 虚线表示忽略液滴蒸发模型中的气相 Soret 扩散, 点 线表示气相甲醇. 从图中可以发现, 甲醇液滴在火焰 前面完全蒸发. 当在蒸发模型中考虑气相的 Soret 扩 散效应时, 液滴表面的甲醇浓度会升高, 这主要是由 于 Soret 扩散会驱动液滴周围的气相向高温区扩散.

在碳氢燃料燃烧过程中, 对碳烟形成的准确模 拟是燃烧模拟的难点之一. 文献 $[25,30]$ 分析了组分 输运模型对同轴射流乙烯扩散燃烧过程碳烟形成的 影响. 在同轴射流装置中, 中间圆管喷射 $32 \%$ 乙烯 $+68 \% \mathrm{~N}_{2}$, 外面同轴圆管喷射空气, 中间圆管的半径 为 $2 \mathrm{~mm}$, 外面同轴圆管半径为 $25 \mathrm{~mm}$, 具体火焰结 构配置读者可以参考文献[30]中的图 2. 图 20 比较了 不同输运模型对乙烯/空气同轴射流扩散火焰中碳烟 体积百分数的影响. 其中图 20(a)中的 M1 代表采用混 合物平均模型计算扩散系数且不考虑 Soret 扩散, M2 代表采用多组分输运模型计算扩散系数且不考虑 Soret 扩散; 图 20(b)中 M3 代表采用多组分输运模型 计算扩散系数且考虑 Soret 扩散. 从图 20(a)中可以发 现, 采用 M1 模型和 M2 模型预测的碳烟体积百分数 基本一致, 且碳烟形成最多的地方在火焰两侧. 图 20(b)比较了 M1 模型和 M3 模型, 可以发现 Soret 扩 散对碳烟形成有显著的影响, 碳烟的位置会整体向 下移动, 且碳烟形成最大位置由火焰侧边移向中心 线. 文献[30]研究表明, 上述现象主要是由大分子燃

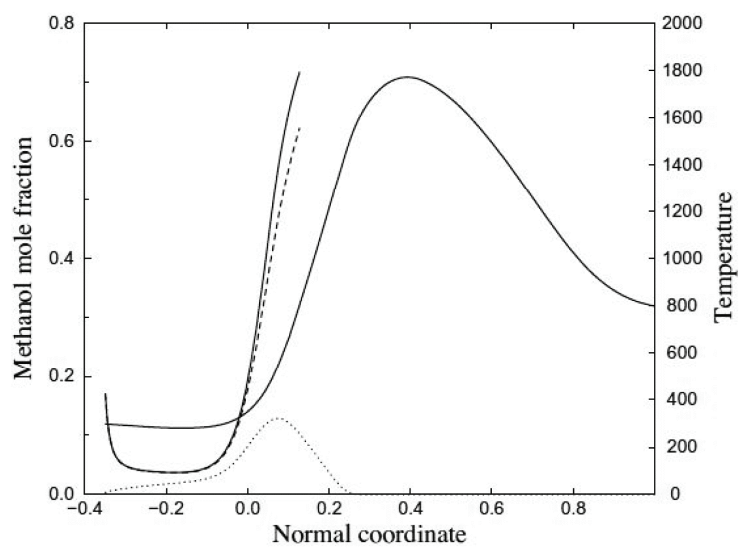

图 19 甲醇喷雾对冲扩散火焰: 甲醇摩尔百分数和温度 $(\mathbf{K})$ 随空间位置的变化 ${ }^{[23]}$
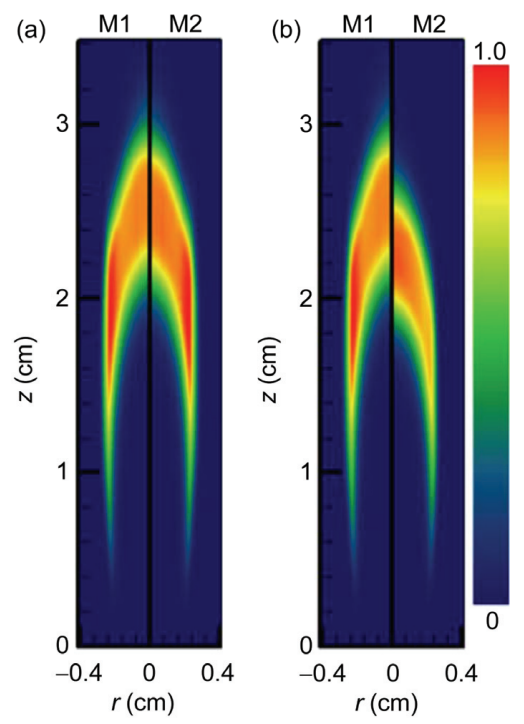

图 20

(网络版彩图)乙烯(a)/空气(b)同轴射流扩散火焰中碳 烟体积百分数 ${ }^{[30]}$

料或自由基的 Soret 扩散引起的.

\section{7 总结与展望}

本文介绍了 Soret 扩散影响层流火焰的相关研究 进展, 回顾了 Soret 扩散对预混火焰、扩散火焰、喷 雾燃烧以及碳烟生成的影响. 关于 Soret 扩散影响层 流火焰的主要结论如下.

对于平面无拉伸火焰, 虽然 Soret 扩散并不会影 响绝热燃烧温度, 但是会改变自由基的输运, 通过输 运与化学反应的耦合作用来影响火焰结构和层流火 焰速度. 而且这种影响会随着当量比、初始温度和初 始压力而变化.

对于拉伸预混火焰, Soret 扩散会与 Lewis 数以及 当量比相结合来改变火焰燃烧强度. 对于轻质燃料, Soret 扩散会增加贫燃火焰的强度和降低富燃火焰的 强度; 而对于大分子燃料, Soret 扩散的作用正好相反. 此外, 当考虑 Soret 扩散时, 火焰对拉伸率的变得更 加敏感; Soret 扩散会降低轻质燃料的最小点火能而 增加大分子燃料的最小点火能.

对于扩散火焰, Soret 扩散会增加轻质燃料的火 焰强度和降低大分子燃料的火焰强度, 从而相应地 改变熄火拉伸率. 另外, 相比于预混对冲火焰, Soret 扩散对扩散对冲火焰熄火拉伸率的影响要更强.

Soret 扩散在喷雾燃烧中会显著地改变液滴表面 
边界层内的组分扩散, 但并不会影响蒸发速率. 大分子燃料和自由基的 Soret 扩散会影响碳烟形 成的位置和数量.

目前对于 Soret 扩散影响各种燃烧系统的认识 尚不完全, 作者认为还有以下几个方面的研究亟待 开展.

(1) 现有的研究主要分析了 Soret 扩散对于预混 火焰点火过程的影响, 然而对于扩散火焰点火问题,
扩散过程会有更重要的作用. 因此, Soret 扩散对扩散 火焰点火的影响是一个值得研究的问题.

(2) 在高温高压等超临界化学反应流中, Soret 扩 散作用越发明显 ${ }^{[27,29,37]}$. 因此有必要研究超临界反应 流中 Soret 扩散的影响.

（3）在一些湍流燃烧模型中, 需要基于层流火焰 面构建数据库, 目前缺乏关于 Soret 扩散影响湍流燃 烧模型的研究 ${ }^{[26,31]}$.

\section{参考文献}

1 Yu W F, Lin Z Z, Ning X J. Mass dependence of the Soret coefficient for atomic diffusion in condensed matter. Phys Rev E, 2013, 87: 062311

2 Rahman M A, Saghir M Z. Thermodiffusion or Soret effect: Historical review. Int J Heat Mass Transfer, 2014, 73: 693-705

3 Joulin G, Clavin P. Linear stability analysis of nonadiabatic flames: Diffusional-thermal model. Combust Flame, 1979, 35: 139-153

4 Buckmaster J, Clavin P, Liñán A, et al. Combustion theory and modeling. Proc Combust Inst, 2005, 30: 1-19

5 Matalon M. Flame dynamics. Proc Combust Inst, 2009, 32: 57-82

6 Poinsot P, Veynante D. Theoretical and Numerical Combustion. Philadelphia: Edwards, 2003

7 Westbrook C K, Mizobuchi Y, Poinsot T J, et al. Computational combustion. Proc Combust Inst, 2005, 30: 125-157

8 Smooke M D. The computation of laminar flames. Proc Combust Inst, 2013, 34: 65-98

9 Law C K. Combustion Physics. Cambridge: Cambridge University Press, 2006

10 Dixon-Lewis G. Kinetic mechanism, structure and properties of premixed flames in hydrogen-oxygen-nitrogen mixtures. Proc R Soc A, 1979, 292: 45-99

11 Greenberg B. On the prediction of thermal diffusion effects in laminar ne-dimensional flames. Combust Sci Technol, 1980, 24: 83-88

12 Garcia-Ybarra P, Clavin P. Cross-transport effects in nonadiabatic premixed flames. In: Proceedings of the 7th International Colloquium on Gasdynamics of Explosions and Reactive Systems, Minsk, Belarus, 1981. 463-481

13 Garcia-Ybarra P, Ncoli C, Clavin P. Soret and dilution effects on premixed flames. Combust Sci Technol, 1984, 42: 87-109

14 Vajda S, Rabitz H, Yetter R A. Effects of thermal coupling and diffusion on the mechanism of $\mathrm{H}_{2}$ oxidation in steady premixed laminar flames. Combust Flame, 1990, 82: 270-297

15 Gomez A, Rosner D E. Thermophoretic effects on particles in counterflow laminar diffusion flames. Combust Sci Technol, 1993, 89: 335-362

16 da Silva L F F, Deshaies B. The influence of equivalence ratio and Sorêt effecton the ignition of hydrogen-air mixtures in supersonic boundary layers. Symp (Int) Combust, 1994, 25: 29-36

17 Ern A, Giovangigli V, Smooke M D. Numerical study of a three-dimensional chemical vapor deposition reactor with detailed chemistry. J Comput Phys, 1996, 126: 21-39

18 Hancock R D, Schauer F R, Lucht R P, et al. Thermal diffusion effects and vortex-flame interactions in hydrogen jet diffusion flames. Symp (Int) Combust, 1996, 26: 1087-1093

19 Ern A, Giovangigli V. Thermal diffusion effects in hydrogen-air and methane-air flames. Combust Theor Model, 1998, 2: 349-372

20 Ern A, Giovangigli V. Impact of detailed multicomponent transport on planar and counterflow hydrogen/air and methane/air flames. Combust Sci Technol, 1999, 149: 157-181

21 Rosner D E, Israel R S, La Mantia B. "Heavy" species Ludwig-Soret transport effects in air-breathing combustion. Combust Flame, 2000, 123: 547-560

22 Williams B A. Sensitivity of calculated extinction strain rate to molecular transport formulation in nonpremixed counterflow flames. Combust Flame, 2001, 124: 330-333

23 Dakhlia R B, Giovangigli V, Rosner D E. Soret effects in laminar counterflow spray diffusion flames. Combust Theor Model, 2002, 6: 1-17

24 Bongers H, De Goey L P H. The effect of simplified transport modeling on the burning velocity of laminar premixed flames. Combust Sci Technol, 2003, 175: 1915-1928 
25 Guo H, Liu F, Smallwood G J, et al. A numerical investigation of thermal diffusion influence on soot formation in ethylene/air diffusion flames. Int J Comput Fluid D, 2004, 18: 139-151

26 Hilbert R, Tap F, El-Rabii H, et al. Impact of detailed chemistry and transport models on turbulent combustion simulations. Prog Energy Combust Sci, 2004, 30: 61-117

27 Palle S, Miller R. Analysis of high-pressure hydrogen, methane, and heptane laminar diffusion flames: Thermal diffusion factor modeling. Combust Flame, 2007, 151: 581-600

28 Arias-Zugasti M, Rosner D E. Soret transport, unequal diffusivity, and dilution effects on laminar diffusion flame temperatures and positions. Combust Flame, 2008, 153: 33-44

29 Ribert G, Zong N, Yang V, et al. Counterflow diffusion flames of general fluids: Oxygen/hydrogen mixtures. Combust Flame, 2008, 154: 319-330

30 Dworkin S B, Smooke M D, Giovangigli V. The impact of detailed multicomponent transport and thermal diffusion effects on soot formation in ethylene/air flames. Proc Combust Inst, 2009, 32: 1165-1172

31 Grcar J F, Bell J B, Day M S. The Soret effect in naturally propagating, premixed, lean, hydrogen-air flames. Proc Combust Inst, 2009, 32: $1173-1180$

32 Yang F, Law C K, Sung C J, et al. A mechanistic study of Soret diffusion in hydrogen-air flames. Combust Flame, 2010, 157: 192-200

33 Yang F, Zhang H Q, Wang X L. Effects of soret diffusion on the laminar flame speed of n-butane-air mixtures. Proc Combust Inst, 2011, 33: 947-953

34 Fong D, Bechtold J K, Law C K. Asymptotic structure of laminar diffusion flames at high pressure. Phys Fluids, 2012, 24: 093602

35 Xin Y, Sung C J, Law C K. A mechanistic evaluation of Soret diffusion in heptane/air flames. Combust Flame, 2012, 159: 2345-2351

36 Zhang H, Yang F, Wang X. The effects of soret diffusion on extinction limit in premixed and diffusion counterflow n-butane/air flames. Combust Sci Technol, 2012, 184: 517-532

37 Liang W, Chen Z, Yang F, et al. Effects of Soret diffusion on the laminar flame speed and Markstein length of syngas/air mixtures. Proc Combust Inst, 2013, 34: 695-702

38 Liang W, Law C K, Chen Z. Role of Soret diffusion in the ignition of hydrogen/air mixtures by a heated kernel. In: 8th US National Combustion Meeting, Park City, UT, USA, 2013. Paper \# 070LT-0030

39 Meynet N, Bentaïb A, Giovangigli V. Impact of oxygen starvation on operation and potential gas-phase ignition of passive auto-catalytic recombiners. Combust Flame, 2014, 161: 2192-2202

40 Sánchez A L, Williams F A. Recent advances in understanding of flammability characteristics of hydrogen. Prog Energy Combust Sci, 2014, 41: $1-55$

41 Giovangigli V. Multicomponent transport in laminar flames. Proc Combust Inst, 2015, 35: 625-637

42 Han W, Chen Z. Effects of Soret diffusion on spherical flame initiation and propagation. Int J Heat Mass Transfer, 2015, 82: 309-315

43 Han W, Chen Z. Effects of soret diffusion on premixed counterflow flames. Combust Sci Technol, 2015, 187: 1195-207

44 Brown N J, Bastien L A J, Price P N. Transport properties for combustion modeling. Prog Energy Combust Sci, 2011, 37: 565-582

45 Williams F A. Combustion Theory. 2nd ed. Menlo Park, CA: Benjamin-Cummins, 1985

46 Kee R J, Grcar J F, Smooke M D, et al. PREMIX: A program for modeling steady, laminar, one-dimensional, premixed flames. Sandia National Laboratory Report SAND85-8240, 1985

47 Chen Z, Burke M P, Ju Y. Effects of Lewis number and ignition energy on the determination of laminar flame speed using propagating spherical flames. Proc Combust Inst, 2009, 32: 1253-1260

48 Chen Z. Effects of radiation and compression on propagating spherical flames of methane/air mixtures near the lean flammability limit. Combust Flame, 2010, 157: 2267-2276

49 Chen Z. On the accuracy of laminar flame speeds measured from outwardly propagating spherical flames: Methane/air at normal temperature and pressure. Combust Flame, 2015, 162: 2442-2453

50 Dai $\mathrm{P}$, Chen Z, Chen S, et al. Numerical experiments on reaction front propagation in n-heptane/air mixture with temperature gradient. Proc Combust Inst, 2015, 35: 3045-3052

51 Kee R J, Miller J A, Evans G H, et al. A computational model of the structure and extinction of strained, opposed flow, premixed methane-air flames. Symp (Int) Combust, 1989, 22: 1479-1494

52 Chen Z, Ju Y. Combined effects of curvature, radiation, and stretch on the extinction of premixed tubular flames. Int J Heat Mass Transfer, 2008, 51: 6118-6125

53 Zhang H, Chen Z. Spherical flame initiation and propagation with thermally sensitive intermediate kinetics. Combust Flame, 2011, 158: 
$1520-1531$

54 Clavin P. Dynamic behavior of premixed flame fronts in laminar and turbulent flows. Prog Energy Combust Sci, 1985, 11: 1-59

55 Zhang H, Guo P, Chen Z. Critical condition for the ignition of reactant mixture by radical deposition. Proc Combust Inst, 2013, 34 : 3267-3275

56 Gopalakrishnan V, Abraham J. Effects of multicomponent diffusion on predicted ignition characteristics of an n-heptane diffusion flame. Combust Flame, 2004, 136: 557-566

\title{
Effects of Soret diffusion on laminar flames
}

\author{
HAN Wang \& CHEN Zheng \\ Department of Mechanics and Engineering Science, College of Engineering, Peking University, Beijing 100871, China
}

Mass diffusion plays a critical role in laminar flames. It affects and even controls flame propagation, ignition and extinction. Usually Ficikan diffusion driven by concentration gradient is dominant while Soret diffusion driven by temperature gradient is relatively small. Therefore, in theoretical analysis and numerical simulation, only Fickian diffusion is considered and Soret diffusion is neglected. However, previous studies indicated that Soret diffusion cannot be neglected for cases with sufficiently light or heavy species and strong temperature gradients. This paper reviews the effects of Soret diffusion on laminar flames. Specifically, we discuss the influence of Soret diffusion on (1) flame propagation, ignition and extinction of premixed planar flames and stretched flames, (2) flame structure and extinction of non-premixed flames, and (3) spray combustion and soot formation.

Soret diffusion, laminar premixed flames, laminar diffusion flames

doi: 10.1360/N092015-00221 